\title{
Un Acercamiento A La Relación Pitagórica A Través Del Cálculo De Ternas
}

\author{
Dr. Carlos Rondero Guerrero \\ M. en C. Marcos Campos Nava
}

Profesor de Tiempo Completo en el Área Académica de Matemáticas y

Física de la Universidad Autónoma del Estado de Hidalgo, México.

\section{M. en C. Agustín Alfredo Torres Rodríguez}

Profesor del Departamento de Ciencias Básicas del Tecnológico Nacional de

México Plantel Atitalaquia, México.

Dr. Juan Alberto Acosta Hernández

Profesor de Tiempo Completo en el Área Académica de Matemáticas y

Física de la Universidad Autónoma del Estado de Hidalgo, México.

\section{Doi: 10.19044/esj.2018.v14n6p58 $\quad$ URL:http://dx.doi.org/10.19044/esj.2018.v14n6p58}

\begin{abstract}
The Pythagorean Theorem and more formally the Pythagorean Relationship (PR), is one of the most well-known and used results in the context of school mathematics, given that it is an axis of conceptual articulation between different areas of Mathematics. However, in the school context it is common for a Didactic Reductionism to be presented, only enunciating it and presenting the algebraic expression that relates the squares of the sides of a right triangle, leaving aside its great historical and epistemological significance. In view of this problem, this documentary research aims to design an alternative proposal to broaden the understanding of the Pythagorean Relationship, which is based on the calculation of Pythagorean Ternas. Three different methods are proposed to generate positive integers, that satisfy the Pythagorean Theorem and suggest questions that can guide learning activities to promote the understanding of some important elements within Mathematics, particularly in reference to the identification of numerical patterns.
\end{abstract}

Keywords: Pythagorean Relationship, Pythagorean triads, Didactic reductionism, Learning Tasks

\section{Resumen}

El Teorema de Pitágoras y más formalmente la Relación Pitagórica (RP), es uno de los resultados más conocidos y utilizados en el contexto de la 
matemática escolar, dado que es un eje de articulación conceptual entre diferentes áreas de la Matemática. Sin embargo, en el contexto escolar es común que se presente un Reduccionismo Didáctico, al sólo enunciarlo y presentar la expresión algebraica que relaciona los cuadrados de los lados de un triángulo rectángulo dejando de lado su gran trascendencia histórica y epistemológica. Ante tal problemática, esta investigación de tipo documental que tiene por objetivo el diseñar una propuesta alternativa para ampliar el entendimiento de la Relación Pitagórica, la cual está sustentada en el cálculo de Ternas Pitagóricas. Se proponen tres diferentes métodos para generar números enteros positivos, que satisfacen el Teorema de Pitágoras y se sugieren preguntas que pueden guiar actividades de aprendizaje para promover el entendimiento de algunos elementos importantes dentro de la Matemática, particularmente en referencia a la identificación de patrones numéricos.

Palabras clave: Relación Pitagórica, Ternas Pitagóricas, Reduccionismo didáctico, Actividad de aprendizaje

\section{Introducción}

La forma en que se presenta el Teorema de Pitágoras (TP) en las asignaturas de Matemática de secundaria y bachillerato, tiene las características de un Reduccionismo Didáctico, que se manifiesta en la manera en que es abordado en los libros de texto, muchas veces limitada a la representación algebraica $a^{2}+b^{2}=c^{2}$, despojada de otros significados, $\mathrm{y}$ enunciada usualmente como: En un triángulo rectángulo, la suma de los cuadrados de los catetos a y b, es igual al cuadrado de la hipotenusa c.

Es necesario recalcar que por contraste, en un sentido más amplio la Relación Pitagórica (RP), conlleva una red de conceptos y significados que se estructuran alrededor del Teorema de Pitágoras, y que abarca diferentes diversas áreas dentro de la Matemática, tales como teoría de números, álgebra, geometría, trigonometría, geometría analítica y el cálculo.

El reduccionismo didáctico de la $\mathrm{RP}$, como se menciona en Reyes, Rondero, Acosta, Campos y Torres (2017), tiene implicaciones en la conceptualización reducida de este saber, es decir, pareciera que un número considerable de estudiantes de nivel universitario, se quedan con muy poco de lo aprendido en los cursos de secundaria y bachillerato, sin que haya por tanto, una notoria ganancia cognitiva entre uno y otro nivel educativo. Lo anterior se ve reflejado en las creencias limitadas que tienen respecto a la RP, y también en referencia a su trascendencia y significado dentro de la Matemática.

Adicionalmente, las diferentes interpretaciones de la RP, se presentan descontextualizadas y desarticuladas unas de otras, lo que conlleva por 
supuesto consecuencias en el aprendizaje de los alumnos. Esta visión simplificada o reducida se hace evidente en diversos libros de texto referidos en los programas de estudio de secundaria, bachillerato, y precálculo, donde se incluyen, según sea el caso, temas de geometría, álgebra, trigonometría y geometría analítica (Rondero, Reyes y Campos, 2015).

\section{Justificación}

Interesados en la problemática anteriomente descrita, nos dimos a la tarea de identificar opciones respecto a cómo enseñar la RP por medio de alguna propuesta didáctica alternativa, que los profesores de Matemática, podrían trabajar transversalmente en secundaria, bachillerato e inclusive en el primer semestre de nivel universitario. Identificamos que el tópico de las ternas pitagóricas es poco conocido por los estudiantes, lo cual fue corroborado en el la revisión documental, antes citada, de algunos libros de texto (Rondero, et al, 2015).

Desde nuestra perspectiva, es muy relevante el hecho conocido de que existan referencias a las ternas pitagóricas desde los antiguos babilonios, lo cual quedó de manifiesto en la tablilla de Plimpton No. 322, referida en Boyer (1991), siendo este descubrimiento arqueológico una evidencia de la importancia que esta cultura daba a tales valores numéricos.

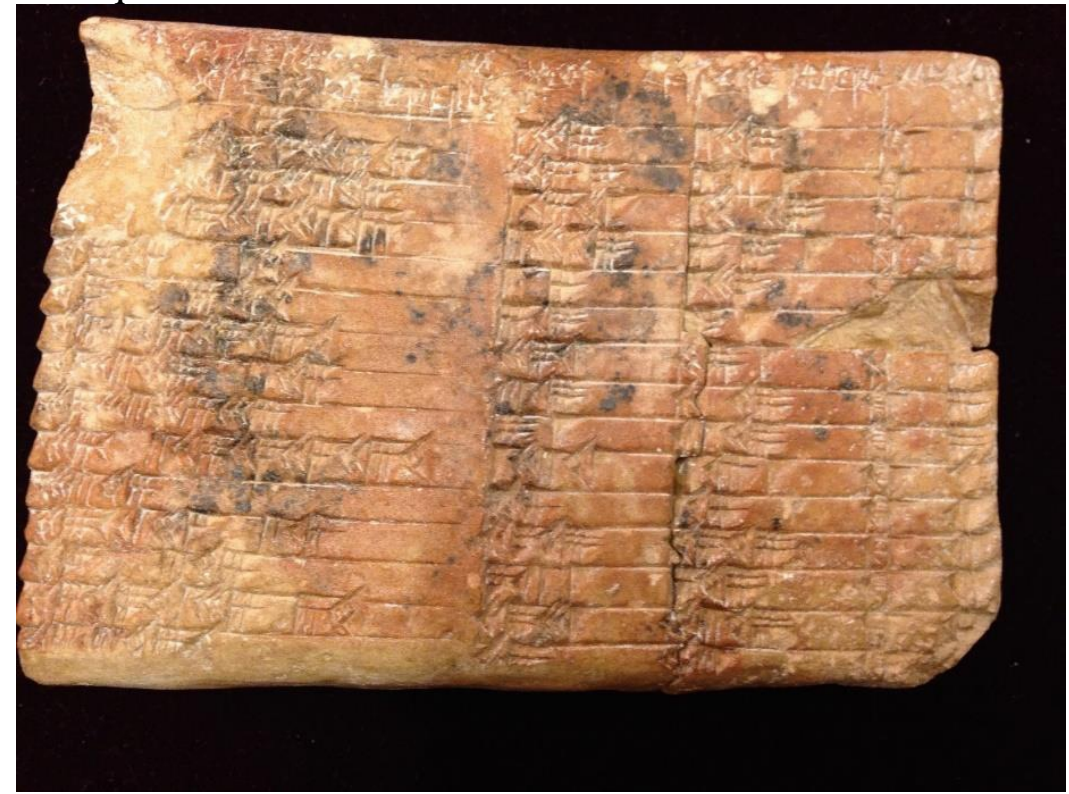

Figura 1. Tablilla Plimpton 322, (aproximadamente 1800 a. d. C.) de la cual se descubrió contiene ternas pitagóricas.

De manera tal, que dada la trascendencia histórica y epistemológica de las ternas pitagóricas, resulta conveniente su incorporación en la didáctica, sobre todo en este caso, con la intención de darle un mayor significado a la 
$\mathrm{RP}$, lo que en principio puede tener incidencias cognitivas en los estudiantes. Partimos de la consideración de que aprender más ampliamente la RP, tiene potencialmente una gran incidencia en la formación matemática de los estudiantes y es por esto que hay necesidad de prestar mucha atención en las formas en que se puede propiciar su acercamiento conceptual y significativo.

\section{Metodología}

Para la realización de este trabajo de investigación nos basamos en un modelo de corte cualitativo, con un diseño no experimental, y de tipo descriptivo. Todo ello debido a que estamos interesados en identificar, a través de una revisión documental, las potencialidades de diversas estrategias didácticas que puedan constituirse en alternativas para lograr una mayor comprensión del tópico de matemáticas en estudio, que en este caso es el de buscar fortalecer los significados en referencia a la RP. Como técnica para el tratamiento de la información recopilada, se utilizó el análisis de contenido, que tiene el propósito de profundizar en la información escrita en forma sistemática y objetiva.

La metodología del análisis de contenido, en el rubro de textos científicos, se efectuó en un nivel descriptivo, con el propósito de descubrir los componentes básicos de un fenómeno determinado, extrayéndolos de un contenido dado (López, 2002). Para nuestros fines, se revisaron documentos diversos sobre métodos de cálculo de ternas pitagóricas, donde lo relevante fue analizar algunas de las ideas centrales de los contenidos, resaltando sus significados, referentes a patrones numéricos y su articulación con otros tópicos como sucesiones, diferencia de cuadrados y media aritmética, entre otros.

\section{Desarrollo}

A continuación se presentan tres diferentes métodos de calcular o generar ternas pitagóricas, con la finalidad de acercar a los estudiantes al estudio de la RP, evidenciando las formas en que es posible encontrar tres números enteros positivos $(n, m, k)$, que cumplen con la igualdad fundamental que relaciona sus cuadrados, $n^{2}+m^{2}=k^{2}$, lo que puede convertirse en una ganancia cognitiva, al ir más allá de sólo quedarse, en el mejor de los casos, con la terna conocida $(3,4,5)$. El cálculo de ternas pitagóricas posibilita, entre otros aspectos, adentrarse en la identificación de patrones numéricos, lo que posteriormente ayuda a entender los procesos de inducción Matemática y además otorga una perspectiva que resulta fundamental en casi cualquier área de la Matemática. En los siguientes métodos intervienen aspectos algebraicos, medias aritméticas, y sucesiones, lo que permite mostrar cómo es posible articular estos temas con la propia RP. 
En este orden de ideas, también nos interesa señalar que, tomando como punto de partida el cálculo de estas ternas, resulta interesante el diseño de actividades de aprendizaje para el aula de Matemática, entendiendo que tales actividades son el vehículo para que los estudiantes aprendan Matemática (Stein y Smith, 1998). Para diversos autores, las actividades que se implementan en el salón de clase, deben diseñarse de forma que "den lugar a procesos inquisitivos de discusión, razonamiento, comunicación y reflexión matemática" (Barrera y Reyes, 2013: 85). En este sentido se tienen coincidencias con enfoques como el de resolución de problemas (Schoenfeld, 1985; Polya, 2005), o el de la construcción de aprendizaje con entendimiento (Hiebert, et al, 2000), para de este modo apoyar un aprendizaje que le proporcione sentido a las ideas y conceptos matemáticos.

El estudio del desarrollo de las ideas, siempre resulta ser revelador de diferentes aspectos epistemológicos, que sin duda tienen diversas implicaciones en los aprendizajes de los conceptos matemáticos. De manera tal, que a continuación se muestran sólo tres formas de calcular ternas pitagóricas, las cuales tienen diferentes características rescatables para la didáctica, en cuanto a significados y articulaciones de saberes.

\section{Métodos de cálculo de ternas}

i) Diferencias de cuadrados igual a otro cuadrado.

Al realizar la diferencia de los cuadrados de dos números naturales consecutivos, se puede identificar un patrón numérico

$$
\begin{gathered}
1^{2}-0^{2}=1 \\
2^{2}-1^{2}=3 \\
3^{2}-2^{2}=5 \\
4^{2}-3^{2}=7 \\
\vdots \\
\cdot \\
(n+1)^{2}-n^{2}=2 n+1
\end{gathered}
$$

La diferencia de cuadrados de dos números naturales consecutivos es un número impar, y si tal diferencia se realiza consecutivamente, se obtiene la sucesión de los números impares.

Por otra parte, al realizar el desarrollo algebraico, como la diferencia de cuadrados igual al producto de binomios conjugados, se obtiene el mismo resultado,

$$
(n+1)^{2}-n^{2}=(n+1+n)(n+1-n)=2 n+1
$$

Es decir, tal diferencia siempre es igual a un número impar $2 n+1$, y en el entendido de que cuando este impar se puede representar como un cuadrado $k^{2}$, entonces se cumple con la RP, tenemos: 


$$
\begin{aligned}
& (n+1)^{2}-n^{2}=k^{2} \\
& n^{2}+k^{2}=(n+1)^{2} .
\end{aligned}
$$

De esta propiedad se pueden calcular ternas pitagóricas, donde claramente dos de los números que la componen son enteros consecutivos, por ejemplo, si $n=4,2 n+1=9=3^{2}$

$$
5^{2}-4^{2}=3^{2} ; \quad 3^{2}+4^{2}=5^{2}
$$

De donde se desprende la conocida terna $(3,4,5)$.

De igual forma, cuando $n=12$, se tiene que $2 n+1=25=5^{2}$, quedando la expresión,

$$
13^{2}-12^{2}=5^{2} ; \quad 5^{2}+12^{2}=13^{2}
$$

En la siguiente tabla se resume el método para generar ternas pitagóricas utilizando diferencias de cuadrados:

\begin{tabular}{|c|c|c|c|c|c|}
\hline $\mathrm{b}=\mathrm{n}$ & $\mathrm{c}=\mathrm{n}+1$ & $2 \mathrm{n}+1$ & $\mathrm{a}=\mathrm{k}$ & $\mathrm{k}^{2}+\mathrm{n}^{2}=(\mathrm{n}+1)^{2}$ & $(\mathrm{k}, \mathrm{n}, \mathrm{n}+1)$ \\
\hline 4 & 5 & 9 & 3 & $3^{2}+4^{2}=5^{2}$ & $(3,4,5)$ \\
\hline 12 & 13 & 25 & 5 & $5^{2}+12^{2}=13^{2}$ & $(5,12,13)$ \\
\hline 24 & 25 & 49 & 7 & $7^{2}+24^{2}=25^{2}$ & $(7,24,25)$ \\
\hline 40 & 41 & 81 & 9 & $9^{2}+40^{2}+=41^{2}$ & $(9,40,41)$ \\
\hline 60 & 61 & 121 & 11 & $11^{2}+60^{2}=61^{2}$ & $(11,60,61)$ \\
\hline
\end{tabular}

Tabla1. Generación de ternas pitagóricas con diferencias de cuadrados

Algunas actividades que se pueden sugerir usando esta tabla, se pueden centrar en la identificación de patrones. Al observar la última columna de la Tabla 1, la que presenta ternas generadas por éste método, el primer término es un impar, el segundo término es un múltiplo de 4, (particularmente de la sucesión de los llamados números triangulares: 1, 3, 6, 10, 15, ..); y el tercero es el consecutivo del segundo. Una vez explicitado este patrón numérico, se puede pedir a los estudiantes que encuentren más ternas pitagóricas, sin necesidad de realizar los cálculos que la Tabla 1 exige.

ii) Números triangulares.

Los números triangulares, identificados por la escuela Pitagórica desde la Grecia antigua, tienen una representación geométrica que permite relacionar un determinado número natural, con el número de puntos que se necesitan para formar cierto tipo triángulos. 

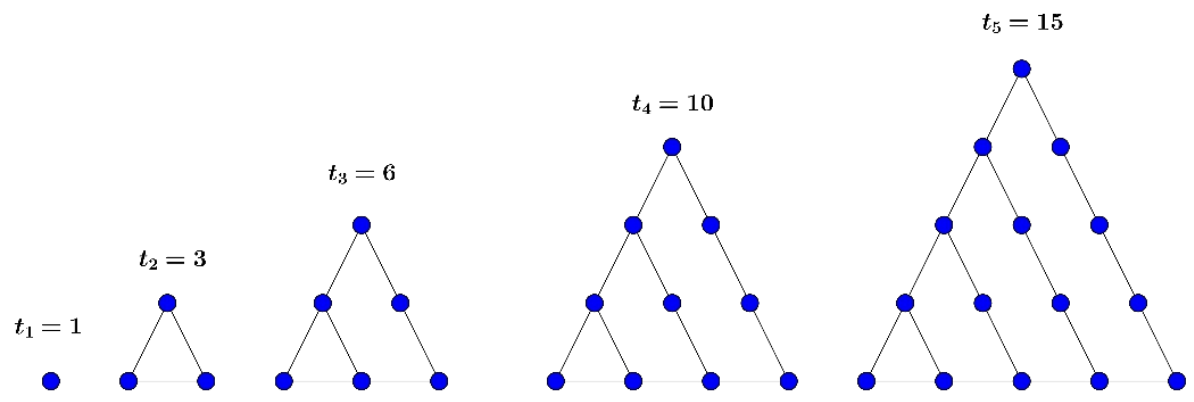

Figura 2. Patrón que siguen los números triangulares.

El patrón como sucesión numérica que siguen estos números se expresa de la siguiente manera:

$$
1,3,6,10,15,21,28,36, \ldots
$$

Si formamos la sucesión de sus diferencias, obtenemos:

$$
1,2,3,4,5,6,7,8, \ldots
$$

Que resulta ser la sucesión de los números naturales, el término nésimo de los números triangulares $t_{n}$, es el resultado de las sumas parciales de $n$ números naturales, por ejemplo, $t_{5}=1+2+3+4+5=15$, de manera general:

$$
t_{n}=1+2+3+4+\cdots+n
$$

Se puede encontrar un patrón numérico que relaciona las medias aritméticas de dos, tres, y hasta $\mathrm{n}$ números naturales consecutivos, con el correspondiente número triangular:

$$
\begin{gathered}
\frac{1+2}{2}=\frac{3}{2} \\
\frac{1+2+3}{3}=\frac{6}{3}=\frac{1+3}{2}=\frac{4}{2} \\
\frac{1+2+3+4}{4}=\frac{10}{4}=\frac{1+4}{2}=\frac{5}{2} \\
\frac{1+2+3+4+5}{5}=\frac{15}{5}=\frac{1+5}{2}=3 \\
\frac{1+2+3+4+5+6}{6}=\frac{21}{6}=\frac{1+6}{2}=\frac{7}{2} \\
: \\
\frac{t_{n}}{n}=\frac{1+n}{2} \Rightarrow t_{n}=\frac{(1+n) n}{2}
\end{gathered}
$$


Esta es la conocida progresión aritmética que permite obtener la suma de $n$ números naturales consecutivos, también conocida como la fórmula de Gauss, donde el n-ésimo número triangular $t_{n}$ es :

$$
1+2+3+4+\cdots+n=\frac{n(n+1)}{2}
$$

Por medio de los números triangulares $t_{n}$, se puede identificar un patrón numérico donde cuatro veces un número triangular, $4 t_{n} \mathrm{y}$ su consecutivo $4 t_{n}+1$, son dos de los elementos de la terna pitagórica, siendo el tercer elemento, el impar correspondiente $2 n+1$. Se puede hacer notar que las ternas calculadas con este método, son las mismas que las obtenidas con el método de diferencia de cuadrados expuesto anteriormente. Este nuevo resultado, se puede utilizar como pretexto para sugerir a los estudiantes como elemento de una tarea de aprendizaje, que se trate de justificar por qué con dos métodos diferentes se pueden obtener las mismas ternas pitagóricas, vale señalar que un elemento el cual diversos autores consideran fundamental en el aprendizaje de las matemáticas, es el poder llegar a un mismo resultado por diferentes métodos. A continuación se presenta en forma tabular, el uso de los números triangulares para generar ternas pitagóricas:

\begin{tabular}{|c|c|c|c|c|c|c|}
\hline $\mathrm{n}$ & $\mathrm{t}_{\mathrm{n}}$ & $\mathrm{a}=2 \mathrm{n}+1$ & $\mathrm{~b}=4 \mathrm{t}_{\mathrm{n}}$ & $\mathrm{c}=4 \mathrm{t}_{\mathrm{n}}+1$ & $\begin{array}{c}(2 \mathrm{n}+1)^{2}+\left(4 \mathrm{t}_{\mathrm{n}}\right)^{2} \\
=\left(4 \mathrm{t}_{\mathrm{n}}+1\right)^{2}\end{array}$ & $\left(2 \mathrm{n}+1,4 \mathrm{t}_{\mathrm{n}}, 4 \mathrm{t}_{\mathrm{n}}+1\right)$ \\
\hline 1 & 1 & 3 & 4 & 5 & $3^{2}+4^{2}=5^{2}$ & $(3,4,5)$ \\
\hline 2 & 3 & 5 & 12 & 13 & $5^{2}+12^{2}=13^{2}$ & $(5,12,13)$ \\
\hline 3 & 6 & 7 & 24 & 25 & $7^{2}+24^{2}=25^{2}$ & $(7,24,25)$ \\
\hline 4 & 10 & 9 & 40 & 41 & $9^{2}+40^{2}+=41^{2}$ & $(9,40,41)$ \\
\hline 5 & 15 & 11 & 60 & 61 & $11^{2}+60^{2}=61^{2}$ & $(11,60,61)$ \\
\hline
\end{tabular}

Tabla 2. Método para generar ternas pitagóricas con números triangulares.

iii) La sucesión de Fibonacci

Es bien conocido el patrón que sigue la sucesión de Fibonacci, la cual se puede construir a partir de un número natural cualquiera. Si iniciamos con el número uno; se repite el primer término de la sucesión y a partir de ahí, se obtiene el siguiente término sumando los dos anteriores, por ejemplo:

$$
1,1,2,3,5,8,13,21,34,55,89 \ldots
$$

También lo son 3, 3, 6, 9, 15, 24, 39,63, .. у 2, 2, 4, 6, 10, 16, 26, 42, 68, .. El patrón de comportamiento de la sucesión de Fibonacci se expresa de la forma,

$$
f_{n}+f_{n+1}=f_{n+2} \quad, \quad n=1,2,3,4,5, \ldots
$$


Algunas de las características de la sucesión de Fibonacci, es que si se toman tres términos consecutivos de ésta, el producto de los extremos y el cuadrado del intermedio se llevan una unidad de diferencia, por ejemplo 2,3,5 verifican que $(2)(5)=10 ; 3^{2}=9 ; 10-9=1$.

A partir de la sucesión de Fibonacci, es posible generar también ternas pitagóricas de la siguiente manera: se toman cuatro términos consecutivos, (i) se multiplican los extremos; (ii) se obtiene el doble producto de los intermedios y (iii) se elevan al cuadrado los intermedios y se suman. En forma tal que los tres números obtenidos en los pasos anteriores, corresponderán a una terna pitagórica, por ejemplo, tomando 1, 1, 2, 3 tenemos: $(1)(3)=3, \ldots$ (i); $2(1)(2)=4 \ldots(i i) ; 1^{2}+2^{2}=5 \ldots(i i i)$; siendo por tanto la terna obtenida $(3,4,5)$. Ahora bien, si se consideran los términos: 1, 2, 3, 5 y repitiendo el mismo procedimiento obtenemos la terna (5, 12, 13), (Kalman y Mena, 2003).

En la siguiente tabla se muestra la construcción de algunas ternas pitagóricas, a partir de la sucesión de Fibonacci.

\begin{tabular}{|c|c|c|c|c|}
\hline $\begin{array}{c}\text { Números de } \\
\text { Fibonacci }\end{array}$ & $\mathrm{a}$ & $\mathrm{b}$ & $\mathrm{c}$ & Terna Pitagórica \\
\hline $1,1,2,3$ & $(1)(3)=3$ & $2(1)(2)=4$ & $1^{2}+2^{2}=5$ & $(3,4,5)$ \\
\hline $1,2,3,5$ & $(1)(5)=5$ & $2(2)(3)=12$ & $2^{2+} 3^{2}=13$ & $(5,12,13)$ \\
\hline $2,3,5,8$ & $(2)(8)=16$ & $2(3)(5)=30$ & $3^{2}+5^{2}=34$ & $(16,30,34)$ \\
\hline $3,5,8,13$ & $(3)(13)=39$ & $2(5)(8)=80$ & $5^{2}+8^{2}=89$ & $(39,80,89)$ \\
\hline $5,8,13,21$ & $(5)(21)=105$ & $2(8)(13)=208$ & $8^{2}+13^{2}=233$ & $(105,208,233)$ \\
\hline
\end{tabular}

Tabla 3. Método para generar ternas pitagóricas con la sucesión de Fibonacci.

Se puede observar que por éste método, las dos primeras ternas $(3,4$, 5) y $(5,12,13)$, son las mismas que se obtienen por los dos métodos descritos anteriormente, el de las diferencias de cuadrados y el de los números triangulares. Sin embargo, las demás ternas que se calculan con éste método, son diferentes a las obtenidas anteriormente, lo cual se puede constatar al comparar las Tablas 1, 2 y 3. Algo que se puede resaltar es el hecho de que salvo las dos primeras ternas, las subsiguientes están formadas por dos enteros que ya no son consecutivos.

Se puede hacer notar la participación relevante que tienen los patrones numéricos y algebraicos, en los métodos descritos, y adicionalmente intervienen en forma articulada temas como diferencia de cuadrados, media aritmética, números triangulares y sucesiones.

\section{Resultados: Sugerencias de actividades de aprendizaje}

Una vez que se tienen identificados los métodos anteriores, pueden constituirse como elementos básicos, para el diseño de actividades de aprendizaje, para ello se pueden considerar las siguientes actividades y cuestionamientos, que tienen por objetivo ampliar la conceptualización y los significados de la RP. 
1. De la Tabla 1, identificando el patrón de comportamiento de los valores correspondientes de $n, m, k$, encontrar las dos siguientes ternas pitagóricas sin necesidad de realizar cálculos que la tabla propone, posteriormente realizar los cálculos identificando el valor de n, comprobar que se cumple la relación

$$
n^{2}+m^{2}=k^{2}
$$

2. ¿En la Tabla 1, es factible encontrar una terna si $n=90$ ? ¿Por qué?

3. De la Tabla 2, identificando el patrón de comportamiento de los números triangulares, que corresponden respectivamente a los valores de $a$, $b, c$, encontrar las dos siguientes ternas pitagóricas y realizar los cálculos identificando el valor de $\mathrm{n}$, para comprobar que se cumple $\mathrm{a}^{2}+b^{2}=c^{2}$.

4. En referencia a la Tabla 2, si $n=20$ ¿es posible encontrar la terna correspondiente? ¿Por qué?

5. ¿Se puede identificar algún patrón numérico en la Tabla 3, que permita encontrar las dos siguientes ternas, sin necesidad de realizar los cálculos que la Tabla propone?

6. Siguiendo el procedimiento de la Tabla 3, usando valores consecutivos de la sucesión de Fibonacci, por ejemplo 8, 13, 21, 34 y 13, 21, 34,55 , encontrar todas las ternas pitagóricas que se pueden generar usando éstos números de Fibonacci.

7. Dada la siguiente terna $(13,84,85)$; comprueba que satisface la RP, identifica de ser posible por cuál de los tres métodos expuestos se generó y describe el procedimiento para obtenerla. (De antemano el estudiante debe entender, que los métodos aquí discutidos no generan todas las ternas pitagóricas posibles).

\section{Reflexiones finales}

-Es muy importante resaltar a los estudiantes la trascendencia que tiene la RP en la estructuración de su alfabetización Matemática, lo que puede redundar en el aprendizaje transversal de las diferentes asignaturas de Matemática, de secundaria, bachillerato y nivel universitario.

-Los tres métodos aquí presentados, para el cálculo de ternas pitagóricas, son sólo algunos de los muchos que se han trabajado en la historia de la Matemática, y consideramos importante su rescate con fines didácticos.

-Además de las ternas, la RP tiene otros contextos de uso dentro y fuera de la Matemática, lo que es una muestra de su trascendencia en la construcción del conocimiento matemático. Es recomendable abocarse a resaltar los contextos, según sea el nivel educativo y la asignatura.

-Un estudio más detallado de la RP, puede propiciar una ganancia cognitiva (Stein y Smith, 1998) por parte de los estudiantes, pero además la identificación de patrones numéricos, que ayuden al desarrollo de su pensamiento matemático. 
-Se requiere impulsar por parte del profesor, una ampliación conceptual de la RP, de manera que lo aquí presentado tiene tal objetivo.

-Las sugerencias de actividades de aprendizaje, aquí presentadas pueden ser trabajadas indistintamente, con algunas variantes, en las asignaturas de matemática de secundaria, bachillerato y precálculo en nivel universitario.

\section{References:}

1. Barrera, F. y Reyes, A. (2013). Elementos Didácticos y Resolución de Problemas: formación docente en matemáticas. México: UAEH Ediciones,

2. Boyer, C. (1991). A History of Mathematics. USA: John Wiley and Sons.

3. Hiebert, J., Carpenter, T. Fennema, E., Fuson, K., Wearne, D., Murray H., Oliver, A. y Human, P. (2000). Making sense. Teaching and learning mathematics with understanding. New Hampshire, USA: Heinemann.

4. Kalman, D. y Mena, R. (2003). The Pythagonacci Family Reunion. Disponible en: http://www.dankalman.net/AUhome/pdffiles/fibpyth.pdf

5. López, F. (2002). El análisis de contenido como método de investigación. Revista Educación, No. 4, pp. 167-179.

6. Polya, G. (2005). Cómo plantear y resolver problemas. México: Editorial Trillas.

7. Reyes, A., Rondero, C., Acosta, J., Campos, M., Torres, A. (2017). Reduccionismo Didáctico y Creencias de Profesores acerca del Teorema de Pitágoras. Bolema: Boletim de Educação Matemática, Vol. 31, No. 59, pp. 968-983.

8. Rondero, C., Reyes, A. y Campos, M. (2015) Análisis de Libros de Texto de Matemáticas de Bachillerato: El Caso de la Relación Pitagórica. En Memorias del VIII Congreso Nacional de Investigación Educativa, COMIE, Chihuahua, México.

9. http://www.comie.org.mx/congreso/memoriaelectronica/v13/doc/132 7.pdf

10. Schoenfeld, A. (1985). Mathematical problem solving. NY, USA: Academic press

11. Stein, M.K. y. Smith, M.S (1998). Mathematical tasks as a framework for Reflection: from Research to practice. Mathematics Teaching in the Middle School, No.3, 268-275. 